THE LANCET,] DR. J. E. SQUIRE : THE SANATORIUM TREATMENT OF CONSUMPTION. [OCT. $28,1905.1239$

hour, the actual figure being influenced by the magnitude of the applied electro-motive force. The amount of zinc set in movement in an ordinary application of ten milliampères for ten minutes is about four milligrammes. The effect of the applications is, therefore, strictly local.

I am not in a position to say anything to-day about the influence of zinc electrolysis upon lupus, although I have tried, and am trying, the method in several cases of the disease. The cases which I chose at the outset for purposes of experiment have not yet proved successful, partly because of their irregular attendances and partly through my own absence from the department during the greater part of July and August, but the partial results so far obtained are not altogether discouraging, and I still hope that an electrolytic method of treating this disease either by zinc (or perhaps by copper) may be obtained. Leduc, in his highly suggestive paper on the "Ions in Medicine," does not by any means limit his remarks to the use of zinc and the treatment of rodent ulcer. He suggests strongly that zinc ions should be tried for all kinds of indolent ulcerations and discharging sinuses by reason of the great antiseptic effect which it exercises. He also deals with the electrolytic introduction of the ions of other metals, of quinine, of salicylic acid, of iodine, and many other drugs, and the impression conveyed by his paper is that, if it can only be established by further experiment that electrolysis provides an effectual mode of percutaneous local medication, its applications in medical practice may become numerous. There is evidently a great deal of work to be done in the development of his ideas. It is historically interesting to recall the fact that Benjamin Ward Richardson made many experiments in the production of local anæsthesia by the introduction of aconite in 1859, but his work met with so much opposition and incredulity that he was forced to give it up. More recently, the discovery of cocaine has enabled the production of local anæsthesia by electrolysis to be carried out with greater success, and I have used this procedure frequently before removing moles and small papillomata by needling.

Edison, in 1890, suggested electrolysis for the introduction of lithium into gouty tissues and since his time several medical experimenters have noted the good effect of this form of treatment. Quite recently Bordier ${ }^{2}$ repeated these experiments, also using as electrode a bath containing a solution of lithium, and made a series of applications to a patient with large gouty deposits in the hands. He was not only able to demonstrate the presence of lithium in the patient's urine, as had been done before by others, but, and this is even more striking, he detected the presence of uric acid in the liquid of the arm baths, thus phoving both the introduction of the kathion lithium and the extraction of the anion uric acid at one operation. There was also a marked change for the better in the condition of the patient's gouty deposits as a result of the experiments. It takes time to follow up and to work out the various lines of research suggested by these new notions of electrolytic medication. The possibilities, as I have endeavoured to show, are highly attractive, but much patient work is still necessary before we can claim anything like final results and I am sure that there is room for many workers in this new line of electro-therapeutic work.

In conclusion, I wish once more to emphasise the fact that the ideas involved in this account of electrolytic medication are the ideas of Professor Leduc of Nantes and that my own position in the development of this line of work is simply that of a follower and disciple of a much esteemed friend.

2 Archives d'Électricité Méđicale, 1900, p. 529.

The Dangers of Anthrax.-The danger involved in working among camel's hair and "low foreign wools" is illustrated by a case that has recently occurred at Bradford. A coroner's jury on Oct. 13th returned a verdict of death from anthrax at an inquiry as to the cause of death of a woman who had been employed as a finisher on camel's hair and low foreign wools. It shows that though these materials " had been sorted, washed, willowed, carded, and combed," the anthrax germs had not been removed nor had they lost their vitality. Something more, therefore, should have been done before those working among them could do so safely, and, moreover, if they had been removed while their vitality remained they would have carried their evil powers with them.

\section{PROBLEM OF HOW BEST TO UTILISE HOSPITALS AND SANATORIUMS IN THE TREATMENT AND PREVENTION OF CONSUMPTION,}

BY J. EDWARD SQUIRE, C.B., M.D. LOND., M.R.C.P. LOND.

SENIOR PHYSICIAN TO THE MOUNT VERNON HOSPITAC FOR DISEASES OF THE CHEST, HAMPSTEAD AND NORTHWOOD.

THERE appears to be a widespread feeling of uncertainty as to whether the sanatorium treatment of consumption has proved sufficiently satisfactory to justify the ever-increasing appeal for funds to maintain existing institutions and to erect new ones. The general public-or such portion of the public as interests itself in such matters-expresses disappointment and hints at failure of the "open-air treatment." The half-penny press, which always knows more than the expert, periodically announces with absolute confi. dence the discovery of some new "cure" for consumption and with equal assurance informs the public later that the supposed cure has proved a failure. Koch's tuberculin, prematurely boomed as a cure for consumption, was condemned as a failure when it was found to do no more than Koch had claimed for it. The hygienic regimen which is found to be so beneficial in the treatment of consumptives was boomed as the "open-air cure." Now that the public is finding out that, though its value fully endorses the expectations of the practical physician, it does not cure all consumptives in a few weeks, it also is condemned as a failure in time to clear the way for the new cure, the conception of which is announced in Paris, the birth being expected to take place in Germany next August. The public holds very strong opinions on medical as on all other subjects-opinions which it expects to have presented to it ready formed each morning with its breakfast. Unfortunately, these "opinions" of ten affect the direction in which the public's charitable gifts are bestowed and it is sometimes necessary that the "opinions" derived from those who assert should be corrected by those who know. It seems that the time has come when those who have experience of the open-air treatment of consumption, who have learned its immense value, and who also know its limitations, should attempt to regulate public opinion on this subject and prevent the swing of the pendulum going too far. Every medical man may be asked to express his views by someone who is convinced that the sanatorium treatment of consumption is a failure but all medical men have not had sufficient personal experience to allow them to speak with conviction. It seems, therefore, desirable that the pros and cons of this most important but somewhat complex problem of the value of sanatorium treatment as a factor in the eradication of consumption should be presented for discussion in the medical journals.

It is unfortunately true that the statistical reports of the various sanatoriums are somewhat misleading, though this is unintentional and to a great extent unavoidable. The improvement undoubtedly shown in many patients when they leave the sanatorium and rightly recorded in the reports may not be maintained when the patient resumes work in unhealthy surroundings; thus the "cure" of one report may subsequently figure as a new admission in another institution. Dr. Ronald Campbell Macfie has recently emphasised the deficiences of such statistical reports and has urged the need of after-care colonies for those "cured" and "improved" whilst in residence in the sanatorium. His article in THE LANCET of Sept. 30th, p. 958, is most valuable in pointing out the limitations of the sanatorium treatment; these limitations must be realised as well as the advantages of the treatment if we would avoid disappointment and escape discouragement. Dr. Macfie states that the sanatorium system is not only medically inefficient but also economically extravagant and he goes on to express the opinion, shared by many, that a careful and successful selection of cases by sanatorium experts would more than double the economic value of sanatoriums. Before we can satisfactorily select the cases, however, we must have a clear idea of what we can hope to achieve by sanatorium treatment. 
The problem which confronts us in attempting to deal with the appalling number of consumptives, with the object of doing the best for the individual sufferer and at the same time endeavouring to stamp out the disease and thus benefit the community, is extremely complex. As visiting physician to the Mount Vernon Consumption Hospital country branch at Northwood this problem has been continually before me during the past year, and with a view to assist the committee of management to a clearer conception of the difficulties which it is so energetically attempting to overcome I sketched a report ${ }^{2}$ which appears to me to serve as a corollary to the article by Dr. Macfie on Sanatoriums for the Poor and the Eradication of Consumption, to which I have already referred. This reports deals with a hospital having various departments and, though reserved for diseases of the chest, not restricted to the treatment of consumption in its early stages. The problem of the utilisation of the hospital to the best advantage of the very large number of consumptives who seek its aid is, however, exactly that with which every sanatorium for consumptives is confronted and the difficulties which present themselves in administration and the management of the patients at Northwood are probably met with in greater or less degree in all sanatoriums. I hardly need then to make any apology for giving the report which follows without any material omission or alteration.

The magnificent gift of the branch hospital at Northwood imposes upon the committee of management and the medical staff of the Mount Vernon Hospital the obligation of utilising this exceptionally fine institution to the best advantage of the greatest number of sufferers from consumption and of the community at large. The utilisation of the hospital to the greatest advantage offers for solution a problem of great magnitude and much difficulty, and there is evidence that its magnitude and difficulties are not entirely realised by the public at large nor by the subscribers and are, perhaps, not fully appreciated by the medical profession. A hospital for consumption and diseases of the chest has a wide rôle to fulfil and great responsibilities. It must be prepared to receive and treat sufferers from all kinds of chest complaints, whether acute or chronic: the acute cases to be treated until cure is complete, the more chronic cases to be "patched up" and rendered able to face a further spell of work. The chest hospitals must also take the lead in educating the public in the measures necessary for the prevention of pulmonary tuberculosis and in teaching patients the required precautions to avoid endangering others. The rôle of these special hospitals and the various departments needed to make them complete were sketched by me in opening the discussion on this subject at the International Tuberculosis Congress in 1901.2

With its out-patient department in Fitzroy-square, in the midst of a densely populated district, the hospital at Hampstead in the healthier air of the outskirts of London, and the country branch hospital at Northwood, the Mount Vernon Consumption Hospital approaches more nearly than any other chest hospital in London (and perhaps in Europe) to the requirements for a complete hospital for diseases of the chest. A colony for the after-care of "cured" consumptives and a home or haven for advanced consumptives are still wanting to make the scheme complete, though the latter requirement is to some extent supplied, so far as concerns the class from which hospital patients are mainly drawn, by the Poor-law infirmaries. The regulations guiding the admission of patients which have been formulated by the medical board serve to indicate the aims and scope of the hospital by showing how provision is made for meeting the needs of the different classes of diseases of the chest for the alleviation and cure of which the hospital is established. It will be seen from these that all kinds of chest diseases may be admitted to the hospital at Hampstead. Some of these-e.g., acute pneumonia-are acute illnesses which require immediate treatment and these may be admitted without waiting as emergency cases. Such cases should require only a short stay in hospital and the aim of treatment is complete cure. A second group comprises heart cases with acute symptoms and the less acute but still curable cases, such as pleurisy, empyema, early tuberculosis, \&c. In these immediate treatment is not so essential, but nevertheless every week of waiting materially affects the

1 This report though drawn up some weeks ago has not yet been presented.

2 Transactions of the British Congress on Tuberculosis, \&c., 1901, vol. ii., p. 443. robability of cure. A third group, by far the largest, comprises the chronic diseases of the lungs and heart, thoracic aneurysms, mediastinal tumours, \&c. In these cases amelioration, not cure, is all that can be expected and in the majority of these cases delay in admission is not of vital consequence.

The hospital at Hampstead must afford help to patients in all these groups and in order that the limited number of beds (about 150) should be available for the largest possible number of sufferers it is essential that the stay of each patient should be short. Many of the patients, with a stay of a month in hospital, will be so far relieved as to be able to return to work for a longer or shorter period.

The country branch hospital at Northwood (100 beds), on the other hand, is not intended for the admission of such a wide range of patients. Its objects are, first, the more prolonged treatment of such cases of consumption as may be curable and, secondly, to provide a short period of rest with country air to convalescents after treatment at Hamp. stead. Not more than one-fourth of the beds at Northwood should be occupied by such convalescents, whose stay should, in the very great majority of cases, not exceed one month. The primary object of the Northwood branch is the curative treatment of consumptive patients and it is with this most important function of the institution, as of all sanatoriums for consumptives, that I propose now to deal. It is now very generally understood that it is in the early stages only of the disease that cure is to be anticipated. This does not imply that the more advanced cases of consumption never become cured; this would be contrary to the experience of all of us, but it is undoubtedly true that, whilst cure may be confidently anticipated if treatment is commenced in the early stages of the disease, the chances of a satisfactory result diminish as the disease advances. It is therefore of the greatest importance that patients with early tuberculosis of the lung should be placed under treatment without delay. Such cases must not be crowded out by chronic cases and thereby be kept waiting for admission. Thus only early cases should be admitted to Northwood or to any sanatorium.

The first difficulty which confronts us in attempting to carry out this essential principle is the want of appreciation of what constitutes an early case. In order to limit to some extent the range of cases which might otherwise be included amongst the "early" cases, the medical board has attempted to fix a standard to which all cases must conform to be eligible for admission to Northwood. It was suggested as a guide to the physicians who examine applicants for admission that, as a rule, selection should be restricted to those in whom the duration of the disease does not exceed 12 months and in whom not more than two lobes of the lung (on the same or opposite side) are affected. This is a very generous interpretation of what constitutes an early case and allows much more mischief than could reasonably be expected to become completely cured. Even with such an elastic definition of an "early" case only a comparatively small proportion of the cases admitted within the past year have come within the limit. This is much to be regretted but is partly attributable to the inevitable difficulty in perfecting the organisation for admission of patients. A similar complaint is to be heard from all sanatoriums.

Amongst the cases which come within the curable limit as to extent and duration of the disease there are different types, with different probabilities as to cure, and it is necessary to observe the course and progress of the illness and to weed out from time to time those cases which do not maintain their favourable character. Even in the most favourable cases the element of time necessary for cure has to be considered. The earliest and most favourable cases will probably require not less than three months to produce quiescence and arrest, and even then it is not possible to speak of the case as "cured." A further period-say from six to 12 months-during which the patient should be kept under observation, must elapse without any evidence of active disease (to insure that the arrest has become permanent) before we can assure the patient that he is cured. In the somewhat more advanced cases cormplete quiescence is improbable under six months, and more likely 12 months may be necessary; even then recrudescence or renewed activity of the disease may occur, so that the patient is not "cured." It is difficult to illustrate this from hospital patients, but amongst private patients $I$ could mention several instances in which such recrudescence recurred occasionally during three or four years before complete arrest of the disease became permanent. Whether such cases ever become: 
"cured" in the sense of gettiug completely free from the seeds of the disease and from the liability to a recrudescence of the mischief is questionable, nevertheless such patients may remain free for the remainder of a long life and are thus practically cured. Still more advanced cases may recover and remain well, but treatment in such cases may have to be continued for several years before arrest is brought about.

There are in London alone about 25,000 consumptives, and the consumption hospitals of the metropolis can provide under 1000 beds altogether, or one bed to every 25 patients, even if only consumptive cases were received and admission were restricted to London patients alone. If each patient were kept on an average three months there would still be only sufficient accommodation for one-sixth of the London consumptives in the year. If we aim at curing patients we must therefore reserve a number of the beds for the curable cases only. In the case of the country branches of the chest hospitals, such as Northwood, and in all sanatoriums this is essential. Some of the curable cases would require from six to 12 months or more for cure to be brought about; it is therefore better when the number of beds is so limited to reserve them for those cases in which arrest might reasonably be expected with three months' treatment in hospital and a period of observation afterwards as outpatients to insure that the arrest remains permanent ("cure").

There is, however, another mode in which the hospital could be usefully employed and by which a greater number of patients could be admitted during the year. If each patient were kept in for one month only this would allow in many cases of the processes which tend towards arrest being commenced, and in all cases it would be long enough to teach the patient how to manage himself so as to prevent the spread of his disease and how to regulate his life to the best advantage to bring about a cure. He could then be discharged to carry on the treatment at home. It is, however, doubtful whether such an extensive and thoroughly equipped institution as the Northwood Hospital is the most suitable place for such educational purpose for poor persons, for the contrast between the hospital and their small, ill-ventilated dwellings would tend to discourage the poorer patients and to make them feel the impossibility of continuing "the cure" in such different surroundings. There is a further objection to restricting the use of the hospital to this purely educational rồle, though in any case the educational value of the hospital is enormous. There is abundance of evidence to show that patients from the poorer districts who have improved considerably under the hygienic conditions of a well-managed consumption hospital or sanatorium may, if they return to their unhealthy surroundings before arrest of the disease is complete, soon relapse; the disease again becomes active and even advances more rapidly than before. In such a case the patient is in a short time worse than if he had never been admitted to hospital and we have done him harm rather than good. It may, therefore, sometimes be kinder and wiser to refuse admission into hospital to a consumptive patient if we are unable to keep him there until his ỏisease has become arrested and, as I have already pointed out, it may take many months for this arrest to be attained. If we are prepared to retain all patients until arrest has occurred we may find that we are only able to treat about two or three patients in the year for each bed in the hospital. This raises at once a very important financial question which materially affects all charitable institutions, though it does not arise in the case of sanatoriums for paying patients. Will persons subscribe annually to an institution if only about 1 in 4 of them can hope to obtain admission for one patient during the year?

The problem is, however, still further complicated when we are dealing with the working classes by the moral effect on the individual of several weeks or months of enforced inactivity. It is found that a large proportion of those patients who remain in the hospital for three months or longer lose all willingness to work ; they even come to object to carrying out the small duties which they at first willingly performed. As their physical capacity for work increases so does their moral distaste for work increase also. We cannot claim to have done the greatest good to the individual if, whilst rendering him physically sound, we have allowed him to degenerate morally. 'To avoid this undesirable result it is advisable that all patients after they have been in residence for three months-or before this if they are fit for it-should have some regular and useful employment provided for them. In this respect, as well as in training patients in healthy occupations, consumptive colonies (or " after-care" colonies) would be most valuable and are much needed. I am informed that at the White Haven Sanatorium, which is worked in conjunction with the Phipps Institute in Philadelphia, every patient has to work for eight hours a day for a month before discharge to insure that he can safely return to work outside.

Such, then, is the problem which it has been our endeavour to solve at Northwood during the last 12 months. As visiting physician it has been my duty to decide upon the length of stay in the hospital for every case and I have felt that the office of visiting physician carries with it a great responsibility. The method which I have adopted in discharging the responsible duties that have been imposed upon me by my colleagues of the medical staff and by the committee of management is as follows: I examine every patient on admission and endeavour to form some opinion as to the probabilities of improvement or of arrest within a reasonable time, noting my conclusions in a special book. Each patient is again brought before me and examined after four weeks' residence; if there does not then seem reasonable expectation of considerable improvement or arrest within three months I disoharge the patient. Where improvement is manifest and likely to continue, even though complete arrest does not seem probable within a reasonable time, I keep the patient in hospital and examine again at the end of another week. Those patients who are steadily progressing towards arrest or cure are not examined again by me for another month. At each examination this "weeding. out" process takes place, no patient being discharged so long as the possibility of cure or arrest within a reasonable time seems hopeful, and, on the other hand, no patient is retained when it seems evident that no further improvement is to be expected within a reasonable time. The definition of the term "reasonable time" is purely arbitrary-the result of my own judgment, taking into consideration on the one hand the probable advantage to the patient of a longer stay and on the other hand the obligations of the institution to those who are waiting for admission. The result is of course a compromise, the majority of the patients being discharged very much improved (and many of them well on the way towards arrest) but yet not "cured," and all having been taught how to regulate their lives to the best advantage. Comparatively few have as yet been kept until the disease has become entirely arrested-time alone can prove whether these have been cured. It should be noted that though the discharge of patients, and thus the duration of the stay in hospital of each one, is left in the hands of the visiting physician the patients are not kept a month without being examined, for besides the resident medical officer there is a resident physician who examines each patient every week. Even under the scheme which I have mentioned I examine from 25 to 50 patients at each visit. Though we may not be able to record a large number of cures as the result of a year's work at the Northwood Hospital, I am satisfied that the institution has been of inestimable value to the majority of those who have been admitted. Many have been able to return to work, some of whom will in all probability remain well for many useful years. Even the few who from the advanced stage of the disease have become worse instead of better whilst at Northwood have at least been made more comfortable than they could possibly have been at home or in any institution in the heart of London. The sanatorium treatment of consumption may not do all that we could wish or fulfil the uninformed anticipations of the man in the street but it is emphatically "worth while." Harley-street, $w$.

\section{THE "LIGHT TREATMENT" OF LUPUS VULGARIS. ${ }^{1}$}

BY MALCOLM A. MORRIS, F.R.C.S. EDIN.,

CONSULIING SURGEON, DRRARIIIENT OF SKMN DISEASE, ST. MARY'S HOSPITAL, LONDON.

THE term "light treatment" is often loosely used, being made to include not only the application of the chemical rays of light concentrated in the manner devised by Finsen but the therapeutic employment of the $\mathrm{x}$ rays. This is misleading, inasmuch as the latter have nothing to do with light

1 A paper read at the International Congress on Tuberculosis at Paris on Oct. 3rd, 1905 . 